\title{
A propos de la détermination de l'angle de frottement des sols pulvérulents au pressiomètre
}

\section{O. COMBARIEU}

Laboratoire régional des Ponts et chaussées

de Rouen, BP 245, 76121 Le Grand Quevilly,

Cedex

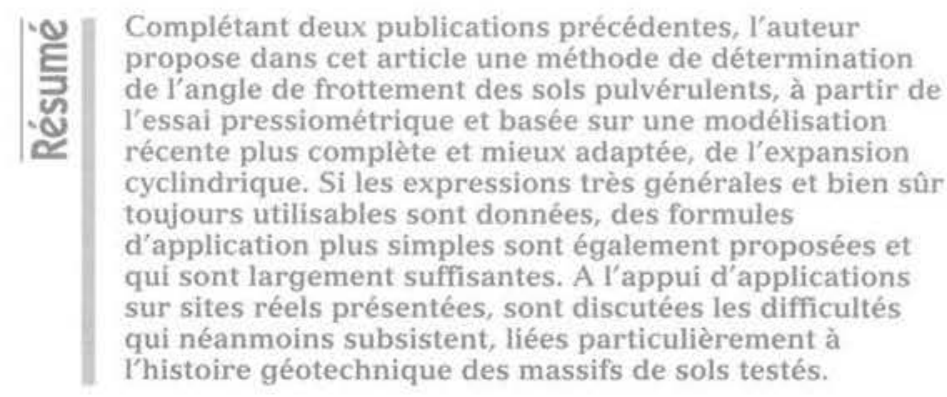

\section{About determination of angle of internal friction of cohesionless soils, with pressuremeter}

Completing two previous publications, the author propound a determination method of the angle of internal friction of cohesionless soils, from pressure - meter test; this method is based on more cormplete and adapted cyclindrical expansion model. Usable generals expressions are given, but simplified formulas are also proposed, which are adequate. After presentation of examples on sites, difficulties are examined, especially resulting from geotechnical chronology of tested soils. 
Cet article constitue un complément à ceux qui ont été récemment publiés dans les Bulletins des Laboratoires des Ponts et Chaussées nos 196 [1] et 203 [2]. On sera tenté de le considérer comme une répétition de ces derniers, mais en réalité il explique les très grandes difficultés, voire les impossibilités, rencontrées jusqu'à récemment, pour déduire de l'essai pressiométrique l'angle de frottement interne $\varphi$ d'un sol pulvérulent ce qui s'avère maintenant plus simple.

Il était en effet étonnant, alors que différents auteurs ont fourni des tableaux ou abaques, très utilisés à l'étranger, donnant $\varphi$ en fonction de la résistance de pointe $q_{c}$ au pénétromètre statique ou de la valeur $\mathrm{N}$ au pénétromètre dynamique SPT, que l'on ne puisse de la même manière obtenir $\varphi$ à partir de l'essai pressiométrique; cet essai est en effet plus riche d'informations que les deux essais précédents et bénéficie, contrairement aux autres, de formulations théoriques reliant les différentes caractéristiques du sol. Il est vrai qu'une relation totalement expérimentale entre l'angle de frottement mesuré à l'essai triaxial et la pression limite pressiométrique a été proposée par Gambin en 1977 [3], relation qui est néanmoins dans bien des cas loin d'être vérifiée.

Cependant, si une meilleure modélisation de la sollicitation pressiométrique permet maintenant cette approche, certains obstacles demeurent; aussi examine-t-on ci-après, les diverses étapes théoriques et pratiques de la démarche.

Au préalable, on rappelle succinctement les hypothèses:

- le sol à un niveau $z$, possède dans le domaine élastique un module d'élasticité isotrope $\mathrm{E}$, lequel est assimilé au module déduit de la courbe de rechargement dans un essai pressiométrique cyclique; le coefficient de Poisson $v$ est de 0,33 ;

- C et $\varphi$, sont les caractéristiques de résistance au cisaillement (critère de Mohr - Coulomb); l'angle de dilatance $\psi$ est la différence entre l'angle de frottement interne $\varphi$, et l'angle de frottement $\varphi$ à l'état critique (cisaillement sans variation de volume). Cette dilatance entraîne donc des variations de volume définies, entre autres, par Monnet et Khlif [4].

\section{Détermination de la pression limite conventionnelle $p_{1, c}$}

Dans tous les développements qui suivent, les pressions, contraintes sont exprimées en valeurs effectives.

- Le premier modèle théorique date des années 1950 [5 et 6]; il conduit pour les sols purement frottants à l'expression

$$
p_{l_{\infty}}=p_{0}(1+\sin \varphi)\left(\frac{E}{2(1+v) p_{0} \sin \varphi}\right)\left(\frac{\sin \varphi}{1+\sin \varphi}{ }^{\prime},\right.
$$

dans le cadre d'une loi élastoplastique simple. $p_{\text {i. }}$ est la pression limite pour une déformation infinie de la sonde de mesure, et $p_{0}$ est la pression horizontale au repos du sol en place. L'essai pressiométrique conduit en fait à mesurer la pression d'expansion pour un doublement du volume initial de la cavité cylindrique; c'est la pression limite conventionnelle, que nous dénommerons ici $p_{t . c}$ avec:

$$
p_{l, c}=p_{o}(1+\sin \varphi)\left(\frac{E}{4(1+v) p_{o} \sin \varphi}\right)^{\left(\frac{\sin \varphi}{1+\sin \varphi}\right)}
$$

E est le module d'élasticité qui peut être mesuré, sur une courbe de second chargement, à l'aide d'un essai pressiométrique cyclique (c'est-à-dire comportant un cycle de déchargement - rechargement); la fixation forfaitaire du coefficient de Poisson v à 0,33 est sans importance, puisque l'essai donne en réalité:

$$
\frac{E}{2(1+v)}
$$

Dans l'intervalle $20^{\circ}<\varphi<50^{\circ}$, (1) peut s'écrire avec une excellente approximation:

$$
\frac{\mathrm{p}_{\mathrm{l}, \mathrm{c}}}{\mathrm{p}_{\mathrm{a}}}=\frac{\pi}{2}\left(\frac{\mathrm{E}}{2 \mathrm{p}_{\mathrm{l}, \mathrm{c}}}\right)^{\sin \varphi}
$$

- Une amélioration importante dans l'expression de $\mathrm{p}_{\text {. }}$ ou $\mathrm{p}_{\mathrm{lc}}$ est obtenue par l'introduction de l'angle de dilatance $\psi$ du sable (qui peut être théoriquement négatif), défini dès 1977 [7] comme la différence entre l'angle de frottement interne $\varphi$ et l'angle de frottement à l'état critique $\varphi$, ce dernier correspondant au cisaillement sans variation de volume. Exprimée alors à la fin des années 1980 [8], on obtient alors:

$$
\mathrm{p}_{\mathrm{l}, c}=\mathrm{p}_{0}(1+\sin \varphi)\left(\frac{\mathrm{E}}{4(1+v) \mathrm{p}_{\mathrm{o}} \sin \varphi(1+\sin \psi)}\right)^{\left(\frac{\sin \varphi}{1+\sin \varphi}\right)(1+\sin \varphi)}
$$

ce qui, pour des mêmes caractéristiques de sol, augmente la valeur de $p_{l, c}$ donnée par (1). L'expression (2) découle de (1) par la simple adjonction du coefficient $(1+\sin \psi)$, aux dénominateur et exposant du terme entre crochet de (1).

$$
\mathrm{p}_{\mathrm{t}, \mathrm{s}} \mathrm{s}^{\prime} \text { écrit aussi pour } 20^{\circ}<\varphi<50^{\circ} \text { : }
$$

$$
\left(\frac{\mathrm{p}_{1, c}}{\mathrm{p}_{\mathrm{c}}}\right)^{11-\sin \alpha \sin \varphi)}=\left(\frac{\pi}{2}-2 \sin \psi \sin \varphi\right)\left(\frac{\mathrm{E}}{2 \mathrm{p}_{\mathrm{l}, \mathrm{c}}}\right)^{(1)+\sin \psi) \sin \varphi}
$$

- Enfin une troisième étape, esquissée partiellement en 1977 [7], puis développée tout récemment [4 et 1], introduit le rôle de la profondeur $\mathrm{z}$, dont il est montré qu'elle peut contribuer à une double plastification du sol lors de la sollicitation pressiométrique. Deux expressions alors s'imposent pour la pression limite: - celle, déjà indiquée en (2) ou ( 2 bis) qui ne s'avère applicable que lorsque $\mathrm{K}_{0}(1+\sin \varphi)>1$;

- et une nouvelle, valable lorsque $K_{o}(1+\sin \varphi)<1$, et qui s'écrit (avec $\mathrm{p}_{\mathrm{o}}=\mathrm{K}_{\mathrm{o}} \mathrm{q}_{\mathrm{o}}$ ):

$$
\frac{p_{1, c}}{q_{0}}=\left(\frac{E(1+\sin \varphi)}{4(1+v) q_{0} \sin \varphi(1+\sin \psi)}\right)^{\frac{\sin \varphi}{1+\sin \varphi}(1+\sin \psi)}
$$

pouvant également s'écrire, toujours pour $20^{\circ}<\varphi<50$ :

$$
\begin{aligned}
& \left(\frac{p_{1, c}}{q_{0}}(1+\sin \varphi)\right)^{[1-\sin \psi \sin \varphi)} \\
= & \left.\left(\frac{p}{2}-2 \sin \psi \sin \varphi\right)\right)\left(\frac{E}{2 p_{1 . c}}\right)^{(1+\sin \varphi) \sin \varphi}
\end{aligned}
$$


On passe simplement de (2) à (3) par le changement de $p_{0}$ en $q_{0}$ et le passage dans le crochet du coefficient $(1+\sin \varphi)$. L'expression (3) peut modifier considérablement, en les augmentant, les valeurs de $p_{1}$ données par (2); elle ne contient pas le coefficient $K_{a}$, et la valeur de $\mathrm{p}_{\mathrm{l}, \mathrm{c}^{\prime}}$ tous paramètres fixés par ailleurs, est donc indépendante de $K_{0}$, tant que $K_{0}<\frac{1}{1+\sin \varphi}$; c'est précisément cette condition que remplissent la plupart des sols granulaires normalement consolidés, a fortiori si l'on admet pour ceux-ci $K_{0}=1-\sin \varphi$, ce qui n'est pas cependant toujours vérifié.

\section{- Application théorique numérique}

On choisira deux massifs de sable; le premier est constitué de sable compact noyé, de poids volumique $\gamma^{\prime}=9 \mathrm{kN} / \mathrm{m}^{3}$, d'angles de frottement $\varphi=40^{\circ}$ et de dilatance $\psi=7^{\circ}$, et pour $z=10$ mètres, de module $E=$ $100 \mathrm{MPa}$; on donnera successivement à $\mathrm{K}_{\alpha}$ les valeurs $0,2,0,3$ et 0,4 .

Le second massif également noyé est constitué de sable lâche, avec $\gamma^{\circ}=6 \mathrm{kN} / \mathrm{m}^{3} \varphi=30^{\circ} \psi=0^{\circ}$ (sable à l'état critique), $\mathrm{E}$ à $\mathrm{z}=10$ mètres vaut $30 \mathrm{MPa}$ avec $\mathrm{K}_{0}=0,45$ ou 0,7 .

Les tableaux I et II donnent, les valeurs de $\mathrm{p}_{1, c}$ en $\mathrm{kPa}$ fournies successivement par (1), (2) et (3); en grisé figure la valeur théorique qui doit être attribuée au massif.

TABLEAU1 Pression limite conventionnelle $\mathrm{p}_{\mathrm{t}}(\mathrm{kPa})$ d'après les formules (1), (2) et (3). Conventional limit pressure $p_{\text {f }}$ from formulas (1), (2) and (3),

Sable compact $E=100 \mathrm{MPa} \quad \varphi=40^{\circ} \quad \psi=7^{\circ} \quad \gamma^{\prime}=9 \mathrm{kN} / \mathrm{m}^{2}$

\begin{tabular}{l|c|c|c}
\hline & $(1)$ & $(2)$ & $(3)$ \\
\hline$K_{0}=0,2$ & 535 & 725 & 1350 \\
\hline$K_{0}=0,3$ & 685 & 905 & 1350 \\
\hline$K_{0}=0,4$ & 815 & 1065 & 1350 \\
\hline
\end{tabular}

Dans cet exemple l'expression (3) est celle qu'il convient d'appliquer, $\operatorname{car} \mathrm{K}_{0}(1+\sin \varphi)$ est toujours inférieur à 1 pour le massif considéré

On peut juger de la différence entre les valeurs réelles, données par (3) et indépendantes de $K_{\text {, }}$ et celles, anciennement données par (1), voire (2) et notoirement plus basses.

TABLEAU III Pression limite conventionnelle $\mathrm{p}_{\mathrm{Hc}}(\mathrm{kPa})$ d'après les formules (1), (2) et (3).

Conventional limit pressure $p_{t,}$ from formulas (1), (2) and (3).

Sable lâche $E=30 \mathrm{MPa} \quad \varphi_{1}=30^{\circ} \quad \psi=0^{\circ} \quad \gamma^{\prime}=6 \mathrm{kN} / \mathrm{m}^{3}$

\begin{tabular}{l|c|c|c|c}
\cline { 2 - 4 } & $(1)$ & $(2)=(1)$ & $(3)$ \\
\hline$K_{e}=0,45$ & 305 & 305 & 395 & $K_{\mathrm{a}}(1+\sin \varphi)=06$ \\
\hline$K_{\mathrm{o}}=0,7$ & 405 & 405 & 395 & $K_{\mathrm{o}}(1+\sin \varphi)=1,05$ \\
\hline
\end{tabular}

Dans le cas de ce massif, les valeurs obtenues de $p_{\text {. }}$ sont faibles et les différences relatives n'atteignent que $30 \%$ d'écart, si $\mathrm{K}_{\mathrm{o}}=0,45$, mais sont pratiquement nulles pour $K_{0}=0,7$ puisque le produit $K_{0}(1+\sin \varphi)$ est très proche de 1 ; la dilatance nulle entraine évidemment $(1)=(2)$.

\section{2}

\section{Détermination de l'angle de frottement $\varphi$}

On imagine aisément que la détermination de I'angle de frottement $\varphi$ (tableau III), problème inverse du précédent, passe par le choix du bon modèle et la connaissance ou l'appréciation de certains paramètres.

On effectue donc ci-après, le calcul de $\varphi$ à $z=$ 10 mètres à partir des résultats théoriques de $p_{\text {f }}$ : $1350 \mathrm{kPa}$ pour le sable compact, et $\simeq 400 \mathrm{kPa}$ pour le sable lâche, valeurs théoriques de la pression limite régnant dans le massif, réputées être celles mesurées.

On suppose bien sûr connus les paramètres $\varphi(=\varphi$ $-\psi$ ), $\gamma$. E étant mesuré au cours de l'essai, et l'on fait varier $\mathrm{K}_{0}$

TABLEAUIII Angle de frottement $\varphi$ d'après les formules (1), (2) et (3).

Angle of internal friction $\varphi$ from formulas (1), (2) and (3).

Sable compact $E=100 \mathrm{MPa} \varphi_{i}=30^{\circ} \gamma^{\prime}=9 \mathrm{kN} / \mathrm{m}^{3} \mathrm{p}_{1 . c}=1350 \mathrm{kPa}$

\begin{tabular}{l|c|c|c}
\hline$K_{0}=0,2$ & $(1)$ & $(2)$ & $(3)$ \\
\hline$K_{0}=0,3$ & $\begin{array}{c}\text { impossible } \\
(\sin \varphi>1)\end{array}$ & $48^{\circ} 3$ & $40^{\circ}$ \\
\hline$K_{0}=0,4$ & $80^{\circ}$ & $45^{\circ} 6$ & 40 \\
\hline
\end{tabular}

TABLEANN Angle de frottement $\varphi$ d'après les formules (1), (2) et (3).

Angle of internal friction $\varphi$ from formulas (1). (2) and (3).

Sable lache $E=30 \mathrm{MPa} \quad \varphi=30^{\circ} \varphi_{1}=30^{\circ} \quad \gamma^{\prime}=9 \mathrm{kN} / \mathrm{m}^{3} \mathrm{p}_{i s}=400 \mathrm{kPa}$

\begin{tabular}{l|l|c|c} 
& $(1)$ & $(2)=(1)$ & $(3)$ \\
\hline$K_{\circ}=0.45$ & $38^{\circ}$ & $38^{\circ}$ & $30^{\circ}$ \\
\hline$K_{\circ}=0.7$ & $30^{\circ}$ & $30^{\circ}$ & $30^{\circ}$ \\
\hline
\end{tabular}

Le tableau III relatif au sable compact montre $l^{\prime}$ inadaptation du modèle $n^{\circ} 1$, trop simple puisque négligeant la dilatance; le modèle 2, s'il s'avère déjà plus satisfaisant, conduit néanmoins à des valeurs bien trop optimistes de $\varphi$. Le tableau IV concernant le sable lâche est d'interprétation plus délicate mais la confrontation de $K_{0}$ et $(1+\sin \varphi)$ permet d'éliminer les valeurs de $38^{\circ}$.

Il faut cependant examiner les modalités pratiques d'application, plus complexes que le simple calcul numérique effectué sur des exemples théoriques.

- L'identification du matériau est nécessaire, de même que le niveau ou les variations du niveau de la 
nappe. De manière plus complète, le niveau piézométrique d'une nappe en charge d'un massif de sable sous-jacent à un horizon imperméable, doit être déterminé; en effet les termes $\mathrm{q}_{0}$ ou $\mathrm{p}_{0}$ d'une importance évidente, sont exprimés en contraintes effectives. La connaissance de la masse volumique ou, du moins, sa bonne appréciation est requise; pour apprécier sans optimisme l'angle de frottement, on voit qu'il faut mieux légèrement la surestimer.

- La réalisation de l'essai pressiométrique, doit être évidemment conforme à la norme NF P 94-110 en vigueur; il est en particulier indispensable de mesurer $p_{1, c}$. La mesure du module du second chargement, à l'aide d'un essai cyclique, fait l'objet d'une proposition de norme actuellement en discussion. Dans la mesure où l'on se contente d'un essai normalisé, l'estimation de $\varphi$ est bien sûr nettement moins bonne, car elle oblige à une hypothèse sur la valeur de $\mathrm{E}$.

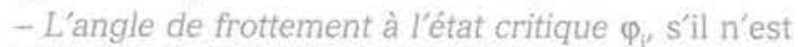
pas connu doit être estimé en fonction de la nature du matériau (nature minérologique des grains, angularité, granulométrie...). A défaut, on pourra adopter une valeur forfaitaire qui a été fixée dans certaines formules pratiques ci-après, à $33^{\circ}$. On trouvera par exemple chez Bolton [9] des éléments utiles à ce sujet; on rappelle néanmoins qu'une détermination de l'angle $\varphi$ est toujours possible en laboratoire, à partir de sable remanié. Une étude est actuellement en cours sur les sables de Honfleur évoqués en 3.

- Enfin, le choix de $K$ se heurte à deux difficultés: il y a lieu d'abord de situer $\mathrm{K}(1+\sin \varphi)$ par rapport à la valeur 1 ; cela conditionne l'application des expressions de type (2) voire (1) ou (3). "L'histoire ) du massif de sol pulvérulent est donc essentielle. Ainsi pour les sols normalement consolidés, on peut légitimement considérer que $K_{0}(1+\sin \varphi)<1$, ce qui facilite grandement le calcul puisque la connaissance précise de $\mathrm{K}$ est alors inutile (expression (3)). Pour les sols qui ont subi des surcharges maintenant disparues, il est moins facile d'apprécier ce critère et encore moins, si $K_{0}(1+\sin \varphi)$ $>1$, de se fixer une valeur réaliste de $K_{0}$, alors nécessaire puisque c'est $p_{0}$ qui intervient (expressions (1) et (2)). Il en est ainsi pour les massifs ayant subi la dernière glaciation, les sols dragués ou les massifs de remblais compactés. Dans la mesure où l'on peut apprécier l'intensité de la surcharge disparue, le sol encore en place ayant mémorisé cette surcharge au niveau des contraintes horizontales $\mathrm{p}_{\alpha}$, on pourra estimer la valeur $\mathrm{K}_{\mathrm{0}, 0 c}$ du sol surconsolidẻ par:

$$
k_{o, o c}=k_{o, n c}\left(\frac{\sigma_{p}^{\prime}}{\sigma_{v o}^{\prime}}\right)^{0.5}
$$

avec, $K_{0 \text { on }}=1-\sin \varphi$ et $\sigma_{\mathrm{p}}^{\prime}$ contrainte de préconsolidation; l'exposant 0,5 qui correspond à $\sin 30^{\circ}$, peut être remplacé par sin $\varphi$; néanmoins cette manière de faire peut être illusoire dans la mesure où $\sigma_{p}^{\prime}$ s'avère souvent difficile à estimer.

On dispose ainsi pour calculer $\varphi$, dans trois situations, de:

1) L'expression (1), pratiquement jamais applicable pour les sols pulvérulents, qu'ils soient normalement consolidés où surconsolidés. Elle implique en effet l'absence de dilatance, donc généralement des sables lâches ainsi qu'un coefficient $\mathrm{K}_{\mathrm{o}}$ relativement élevé, au minimum de l'ordre de 0,7. Cette situation est donc rare ; l'expression (1) n'est autre que l'expression (2) pour $\psi=0$, et exige $K_{0}(1+\sin \varphi)>1$. Notons qu'elle permet par le biais de (1 bis) d'écrire simplement:

$$
\sin \varphi=\frac{\ln \frac{2 \mathrm{p}_{\mathrm{lc}}}{\pi \mathrm{p}_{0}}}{\ln \frac{\mathrm{E}}{2 \mathrm{p}_{\mathrm{l}, \mathrm{c}}}}
$$

2) Lorsque $K_{0}(1+\sin \varphi)>1$, ce qui concerne a priori des sols fortement surconsolidés, et en particulier des sols artificiels compactés, on appliquera (2) ou ( 2 bis) dans son intégralité.

Ces expressions peuvent d'ailleurs dans le domaine $25^{\circ}<\varphi<50^{\circ}$ s'écrire sous la très bonne forme suivante:

$$
\sin \varphi=\frac{\ln \frac{2 p_{l, c}}{\pi p_{0}}}{\ln \frac{E}{2 p_{1 . c}}+\left(\ln \frac{E}{2 p_{0}}-\frac{4}{\pi}\right) \sin \left(\varphi-\varphi_{1}\right)}
$$

facile à résoudre en $\varphi$. Un essai alterné fournira $E$ et $p_{L}$ sachant qu'on pourra sans grand inconvénient se fixer une valeur de $\varphi_{i}$; le plus délicat est d'estimer $\mathrm{K}_{\mathrm{o}^{\prime}}$ donc $\mathrm{p}_{\mathrm{o}}$, qui doit être compatible avec des valeurs vraisemblable de $\varphi$. Ce cas d'application est certainement le plus délicat au niveau de l'interprétation. L'absence d'un essai cyclique rend encore plus délicat la détermination de $\varphi$.

La formule ( 2 ter) est tabulée dans le tableau V suivant, pour des valeurs de $\varphi\left(30^{\circ}-33^{\circ}\right.$ et $\left.36^{\circ}\right)$ et pour $=$ $\mathrm{E} / \mathrm{P}_{\mathrm{ic}}=25-30-50-75-100$, lequel permet, pour les applications, de cerner les marges d'incertitude; un abaque peut être facilement dressé.

3) Lorsque $K_{0}(1+\sin \varphi)<1$, ce qui correspond très généralement aux sols normalement consolidés, mais peut aussi concerner des sols surconsolidés, on pourra appliquer (3) ou (3 bis), dans son intégralité.

La formule (3 bis) n'a pas été tabulée ici. On remarquera simplement qu'un tel tableau serait identique au précédent $n^{\circ} 5$, dans lequel la colonne $\frac{P_{l, c}}{P_{o}}$ serait rem$p_{0}$ placée par $\frac{p_{L, c}}{p_{0}}(1+\sin \varphi)$ ce qui permet très rapidement de trouver $\varphi$ connaissant $\frac{E}{p_{l, c}}, \varphi_{1}$ et $\frac{p_{l, c}}{q_{o}}$.

En outre, nous avons montré [2] que l'on peut écrire $\frac{p_{1, c}}{q_{0}}=a\left(\frac{E}{2 q_{0}}\right)^{\frac{\sin \varphi}{1+\sin \varphi}(1+\sin \psi)}$

avec approximativement:

$$
\begin{aligned}
& a=1,1 \quad \text { pour } \varphi \leq 32^{\circ} \\
& \mathrm{a}=1 \text { pour } 32^{\circ}<\varphi<36^{\circ} \\
& \mathrm{a}=0,9 \text { pour } \varphi \geq 36^{\circ}
\end{aligned}
$$

Avec la réalisation d'un essai cyclique, donnant $\mathrm{E}$, on pourra utiliser l'expression suivante en découlant, qui donne d'excellents résultats: 
Détermination of $\varphi$, d'après la formule (2 ter). Determination of $\varphi$, from formula (2 ter).

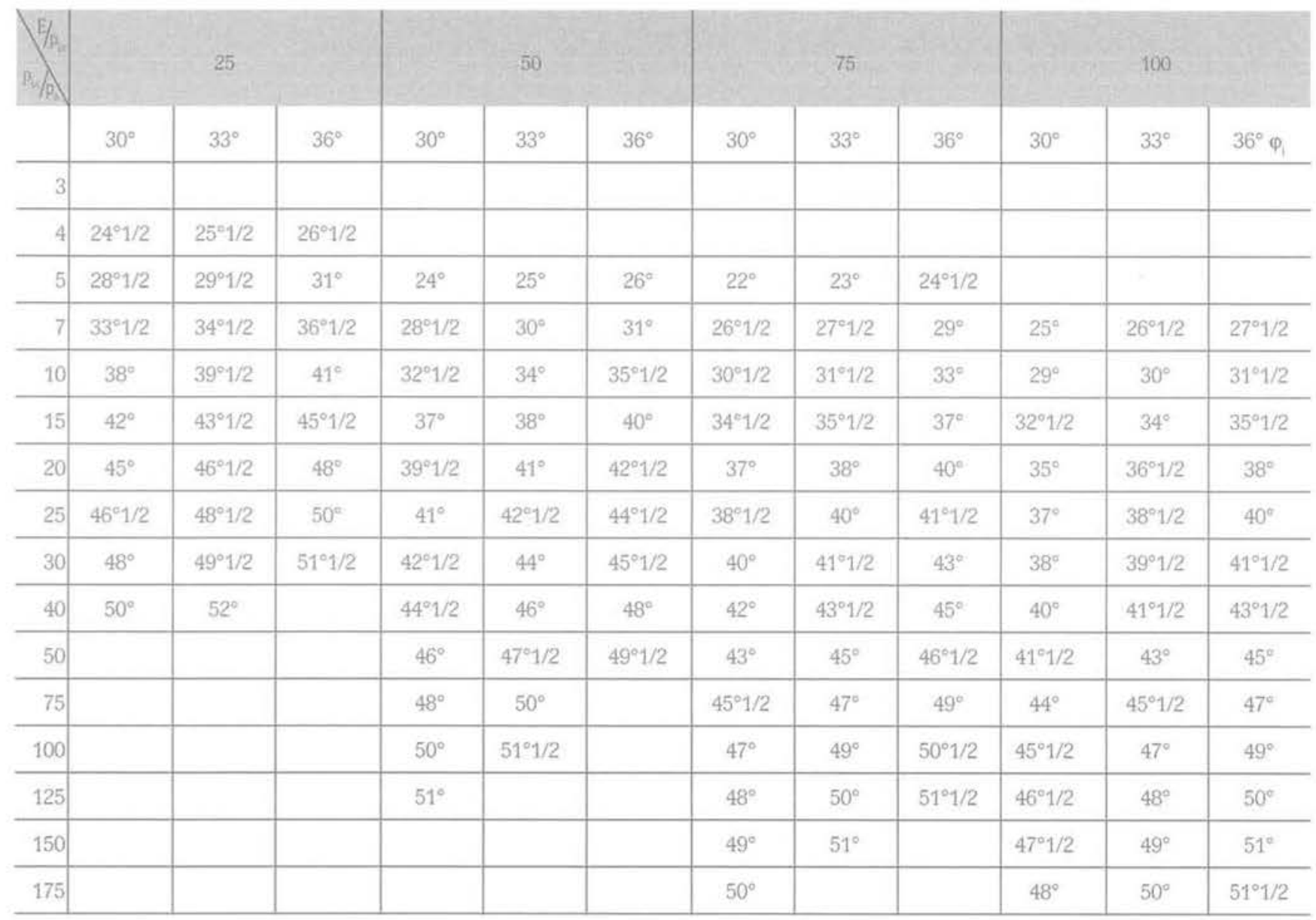

(3 ter)

$$
\sin \varphi=(1+b)\left(b+\frac{\ln \frac{p_{l c}}{a q_{0}}}{\ln \frac{E}{2 q_{0}}}\right)
$$

avec b valant respectivement $\frac{1}{7} ; \frac{1}{8} ; \frac{1}{9}$ pour $\varphi$, valant

$30^{\circ}, 33^{\circ}$ ou $37^{\circ}$ et a, valant 1,$1 ; 1$ et 0,9 quand

$$
\frac{p_{1, c}}{q_{0}^{\prime}} \leq 6 ; 6<\frac{p_{1, c}}{q_{0}^{\prime}}<12 \text { ou } \frac{p_{1, c}}{q_{0}^{\prime}} \geq 12 \text {. }
$$

Dans la mesure où l'on ne réalise qu'un essai normalisé (donc sans cycle), il est évident que l'imprécision sur la détermination de $\varphi$ augmente, et dans ce cas on admettra pour $\Psi=\varphi-\varphi$, la valeur $\varphi \mathrm{i}=33^{\circ}$, a étant arbitrairement choisi égal à 1 . On admet dans ce cas un ordre de grandeur de $\mathrm{E} / \mathrm{p}$, de 50 à 60 .

On propose donc dans ces conditions pour l'essai normalisé où $p_{1}$ a été effectivement mesuré, la formule simplifiée:

$$
\sin \varphi=\frac{9}{8}\left(\frac{1}{8}+\frac{\ln \frac{p_{1, c}}{q_{0}}}{\ln \frac{p_{1, c}}{q_{0}}+\left(\frac{3}{2}\right)^{3}}\right)
$$

(3 quarto)
Pour conclure sur les possibilités de détermination de $\varphi$, celle-ci est d'autant plus précise que les facteurs entrant dans les expressions sont connus. On pourra alors appliquer les expressions complètes (2) ou (3), celles relatives aux sols pulvérulents normalement consolidés (3) présentant l'avantage de ne pas nécessiter la connaissance de $\mathrm{K}_{\mathrm{i}}$

Pour les sols surconsolidés où $K$ peut être très élevé, la difficulté d'interprétation est évidente.

On notera en outre, que dans le cas de sols compactés ou de sols hors nappe, l'existence d'une cohésion capillaire c pertube également les résultats en augmentant la pression limite. Les formules (1), (2) et (3) sont d'ailleurs dans ce cas modifiées, les termes $p_{1, c}, p_{0}$ et $q_{0}$ se trouvant alors remplacés respectivement par $p_{i c}$ $+c \cdot \operatorname{cotg} \varphi, p_{0}+c \cdot \operatorname{cotg} \varphi$ et $q_{0}+c \cdot \operatorname{cotg} \varphi(1+\sin \varphi)$.

\section{Applications numériques}

Celles-ci ont été effectués sur des sites où des essais normalisés ou cycliques ont été réalisés; ils concernent des massifs normalement consolidés ou compactés.

- Sables alluvionnaires de l'estuaire de la Seine, normalement consolidés; ils ont fait l'objet d'essais cycliques à 6, 7 et 8 mètres de profondeur, dans un massif, caractérisé suivant le schéma ci-après: 
terrain naturel (TN), à $0 \mathrm{~m}$

$$
\gamma_{\mathrm{h}}=18 \mathrm{kN} / \mathrm{m}^{3} \quad \varphi \text { triaxial }=35^{\circ}(\text { minimum })
$$

nappe à $1,50 \mathrm{~m}$

$$
\begin{aligned}
& \gamma_{\mathrm{h}}^{\prime}=9,5 \mathrm{kN} / \mathrm{m}^{3} \varphi \text { triaxial }=45^{\circ} \text { (maximum) } \\
& \mathrm{K}_{\mathrm{o}}=0,45
\end{aligned}
$$

à $\mathrm{z}=6 \mathrm{~m} \quad \mathrm{P}_{\mathrm{lc}}=620 \mathrm{kPa} \quad \mathrm{E}=26,5 \mathrm{MPa} \quad \mathrm{q}_{0}^{\prime}=69,75 \mathrm{kPa}$

à $\mathrm{z}=7 \mathrm{~m} \quad \mathrm{p}_{1, \mathrm{c}}=750 \mathrm{kPa} \quad \mathrm{E}=40 \mathrm{MPa} \quad \mathrm{q}_{\mathrm{o}}^{\prime}=79,25 \mathrm{kPa}$

à $\mathrm{z}=8 \mathrm{~m} \quad \mathrm{p}_{\mathrm{l}, \mathrm{c}}=1500 \mathrm{kPa} \quad \mathrm{E}=66,5 \mathrm{MPa} \quad \mathrm{q}_{0}^{\prime}=88,75 \mathrm{kPa}$

L'expression applicable est la $n^{\circ}(3)$ ou ( 3 bis), pour laquelle on a choísi $\varphi=30^{\circ}$, qui conduit pour les trois niveaux examinés aux valeurs de $38^{\circ}, 37^{\circ}, 44^{\circ}$, pour $\varphi$.

Si l'on fait abstraction de l'essai cyclique, l'application de ( 3 ter), conduit à $36^{\circ}, 36^{\circ}, 41^{\circ}$, valeurs légèrement plus faibles et dans le sens de la sécurité.

Les résultats sont en bon accord avec les mesures directes faites à l'appareil triaxial.

\section{- Calais. Étude d'un quai en eau profonde}

Sur ce site, les sables flandriens règnent sur environ $30 \mathrm{~m}$ d'épaisseur, de $9 \mathrm{~m}$ à $-22 \mathrm{~m} \mathrm{CM}$, avec une nappe de niveau moyen $+4,50 \mathrm{~m} \mathrm{CM}$. Leur poids volumique saturé atteint $20 \mathrm{kN} / \mathrm{m}^{3}$, pour environ $18 \mathrm{kN} / \mathrm{m}^{3}$ hors nappe, et des angles $\varphi$ sont compris entre $37^{\circ}$ et $41^{\circ}$ suivant les mesures à la boite de cisaillement ou au triaxial; une légère cohésion, variable suivant les essais, de $6 \mathrm{kPa}$ moyen, existe surtout dans les zones situées hors nappe.

Les diagrammes donnant $p_{k}$ et $E_{M}$ en profondeur, sont traduits par deux droites moyennes

$$
\begin{array}{rlr}
\mathrm{E}(\mathrm{MPa})=10+0,9 \mathrm{z}(\mathrm{m}), & \\
\text { et } \quad \mathrm{P}_{\mathrm{L}, \mathrm{k}}(\mathrm{kPa}) & =800+90 \mathrm{z} & \text { pour } \mathrm{z}<4,5 \mathrm{~m} \\
& =820+85 \mathrm{z} & \text { pour } \mathrm{z}>4,5 \mathrm{~m}
\end{array}
$$

mais une très forte dispersion existe.

Le sable étant normalement consolidé, on a: $\mathrm{K}_{0}(1+\sin \varphi)<1$;

l'application de (3 ter), avec $\mathrm{q}^{\prime}$ ( $(\mathrm{kPa})=18 \mathrm{z}$ pour $\mathrm{z}<4,50 \mathrm{~m}$

et $\mathrm{q}_{\mathrm{o}}^{\prime}(\mathrm{kPa})=4,50 \times 18 \mathrm{z}+(\mathrm{z}-4,5) 10=36+10 \mathrm{z}$ pour $z>4,50 \mathrm{~m}$

donne respectivement, avec une cohésion supposée nulle, pour:

$$
\begin{gathered}
z=3 m \quad \frac{p_{1, c}}{q_{0}^{\prime}}=19,8 \quad \varphi^{\prime}=41,5^{\circ} \\
z=10 m \frac{p_{i, c}}{q_{0}^{\prime}}=12,3 \quad \varphi^{\prime}=38^{\circ} \\
\text { et } z=25 m \frac{p_{i, c}}{q_{o}^{\prime}}=10,3 \quad \varphi^{\prime}=37^{\circ}
\end{gathered}
$$

Toujours sur ce même site, la construction du quai a amené lors d'une phase de travaux, à draguer les sables jusqu'à -4,50 m CM et ensuite un rabattement de nappe important le permettant, à les remettre en place par compactage traditionnel entre $-4,50 \mathrm{~m} \mathrm{CM}$ et 8,00 m CM.

Si ces sables en remblai possèdent des poids volumique et angle de frottement identiques à ceux initiaux en place, on constate par contre des pressions limites nettement plus élevées; le diagramme $\mathrm{p}_{1}(\mathrm{kPa})=200$ $+14 \mathrm{z}(\mathrm{m})$ représente correctement cette variation. Les modules pressiométriques n'échappent pas à cette remarque, puisque $E_{M}(\mathrm{Mpa})=20+1,4 \mathrm{z}(\mathrm{m})$. Toujours avec une nappe à $4,50 \mathrm{~m}$ de profondeur donnant la même variation de $\mathrm{q}^{\prime}$, avec la profondeur, la recherche de $\varphi$, doit s'opérer à l'aide des formules ( 3 ter) ou ( 2 ter) puisque la valeur de $\mathrm{K}$ peut être relativement élevée, du fait du compactage, et l'on ne connaît pas la valeur de $K_{0}(1+\sin )$ par rapport à 1 .

$\mathrm{A} 3,00 \mathrm{~m}$ où $\mathrm{q}_{0}^{\prime}=54 \mathrm{kPa}$ et $\mathrm{p}_{\mathrm{l}, \mathrm{c}}=2420 \mathrm{kPa}$, on trouverait avec ( 3 quarto) $\varphi=47^{\circ}$, ce qui est manifestement trop fort; la formule (3), plus exacte, avec $\mathrm{E} / \mathrm{p}=55$ comme pour ( 3 quarto) donne, elle, $\varphi=50^{\circ}$.

A $10,00 \mathrm{~m}, \mathrm{q}_{0}^{\prime}=136 \mathrm{kPa}$ et $\mathrm{p}_{1 \mathrm{c}}=3400 \mathrm{kPa} \varphi=43,5^{\circ}$ (avec 3 quarto) et $48^{\circ}$ avec (3).

Par contre l'application de (2) ou de (2 ter), pour $3,00 \mathrm{~m}$ et $10,00 \mathrm{~m}$ fournit le tableau VI suivant, en fonction de $\mathrm{K}_{\mathrm{o}}$

TABLEAUY Sables de remblai de Calais: angle $\varphi$ suivant (2) ou ( 2 ter). Calais sands; angle $\varphi$ from (2) or (2 ter).

\begin{tabular}{c|c|c}
\hline$K_{n}$ & $3 \mathrm{~m}$ & $10 \mathrm{~m}$ \\
\hline 0,8 & $47,5^{\circ}$ & \\
\hline 1,2 & $45^{\circ}$ & $43,5^{\circ}$ \\
\hline 1.5 & $43,5^{\circ}$ & $40,5^{\circ}$ \\
\hline
\end{tabular}

S'il apparaît que la valeur de $\mathrm{K}$ est probablement de l'ordre de 1, comme nous l'avons signalé, l'interprétation se révèle délicate et, dans le cas de remblai, nécessite obligatoirement un essai cyclique, la fixation ici à 55 du rapport $\mathrm{E} / \mathrm{p}$ étant arbitraire. De plus, une légère cohésion dans les premiers mètres du terrain influe également sur le résultat.

En l'occurence, puisque le propos est d'estimer $\varphi$, dans le cas présent, en choisissant des valeurs de $\mathrm{K}$ de l'ordre de 1 voire plus, ce qui parait raisonnable, on voit qu'une valeur de $40^{\circ}$ est correcte. Mais il faut reconnaître que deux inconnues subsistent: $\mathrm{K}_{0}$ et $\varphi$.

- Rideau expérimental de Karlsruhe (Allemagne)

Dans le cadre de la normalisation européenne, une expérimentation sur rideau a fait l'objet de différents calculs comparatifs entre pays européens. Le massif de sable, en poussée, derrière le rideau étudié, possède un angle de frottement moyen de $40^{\circ}$ à $41^{\circ}$ mesuré en laboratoire.

L'examen du profil pressiométrique $F_{3}$, réalisé à cet effet par le LRPC de Strasbourg suivant l'essai normalisé, avec une masse volumique de $16 \mathrm{kN} / \mathrm{m}^{3}$, conduit sur $4,50 \mathrm{~m}$, aux valeurs approchées de $\varphi$ suivantes (formule 3 quarto):

$$
\text { à } \begin{array}{ll}
1,00 \mathrm{~m} & 46^{\circ} \\
1,80 \mathrm{~m} & 40^{\circ} \\
2,60 \mathrm{~m} & 42^{\circ} \\
3,40 \mathrm{~m} & 42^{\circ} \\
4,20 \mathrm{~m} & 39^{\circ}
\end{array}
$$

Seule la valeur à $1,00 \mathrm{~m}$ proche de la surface, apparaît très élevée dans cet exemple et peut correspondre à une cohésion non négligeable, ou a un module de déformation particulièrement élevé lié à la proximité de la surface (dessiccation...). 


\section{- Remblai de la déviation de la RN 13 à Lisieux (Calvados)}

Cet important remblai d'accès à un ouvrage d'art est constitué de sable de Glos, qui est un sable extrêmement fin, rappelant les sables de dune, et assez homométrique. Un profil pressiométrique normalisé, réalisé sur 5 mètres, a fourni les valeurs suivantes pour $p_{l, c}$

$\begin{array}{rr}1 \mathrm{~m} & 1210 \mathrm{kPa} \\ 2 \mathrm{~m} & 1020 \mathrm{kPa} \\ 3 \mathrm{~m} & 700 \mathrm{kPa} \\ 4 \mathrm{~m} & 1040 \mathrm{kPa} \\ 5 \mathrm{~m} & 1020 \mathrm{kPa}\end{array}$

On attribue au sable, de poids volumique mesuré, $18 \mathrm{kN} / \mathrm{m}^{3}$, un angle critique $\varphi$ de $30^{\circ}$. En se fixant a priori $\mathrm{K}$ de l'ordre de 1 , et $\mathrm{E} / \mathrm{p}$, de 50 , l'application de (2), conduit respectivement à:

$\begin{array}{ll}1 \mathrm{~m} & 47^{\circ} \\ 2 \mathrm{~m} & 42^{\circ} \\ 3 \mathrm{~m} & 35^{\circ} \\ 4 \mathrm{~m} & 36^{\circ} \\ 5 \mathrm{~m} & 34^{\circ}\end{array}$

à comparer à $41^{\circ}$ mesuré lors d'un unique essai à l'appareil triaxial. On constate comme pour Calais précédemment, que le premier mètre conduit à une valeur très forte. Mais on peut noter que l'absence d'essai normalisé et la méconnaissance de $\mathrm{K}_{0}$ entrainent des incertitudes importantes.

\section{4}

\section{Conclusion générale}

Au terme de cet article, la valeur de l'angle de frottement $\varphi$ des sols pulvérulents apparaît appréhendable à partir de l'essai pressiométrique. Certes, déterminer ainsi $\varphi$ ne reste néanmoins pas si simple et requiert des essais de qualitê, laquelle malheureusement n'est généralement plus la vertu principale de nombreux essais de ce type.

En effet, les «essais alternés », ainsi dénommés dès 1963 par L. Ménard et que la norme à venir appellera cycliques, constituent une difficulté supplémentaire à un essai, quoiqu'on en dise, pourtant déjà délicat. De ce point de vue, et dans le but poursuivi ici, l'accumulation à partir d'essais minutieux de résultats sur les rapports $\mathrm{E} / \mathrm{E}_{\text {, }}$ sur les valeurs de $\mathrm{K}_{\mathrm{o}}$ (à partir d'essais par autoforage ou en laboratoire), et sur les valeurs de $\varphi$ et $\varphi_{\text {i, }}$ en laboratoire, doit permettre de mieux tirer profit du seul essai normalisé.

Demandant un jour à une société de sondages l'exécution d'essais alternés, cette dernière s'étonnait qu'on lui demandât de ne réellement exécuter qu'un essai sur deux... alors qu'il s'agissait pratiquement d'en réaliser deux en un...! Baptiser un tel essai «cyclique » évitera, il faut l'espérer, cette «confusion».

\section{$\overline{\text { Bibliographie }}$}

[1] Combarieu O:- L'essai pressiomètrique et la résistance au cisaillement des sols Bulletin de liaison des $L P C, \mathrm{n}^{\circ} 196, \mathrm{p}, 43$ 51, 1995

[2] Combarieu O. - Estimation rapide de l'angle de frottement d'un sol granulaire au pressiomètre. Bulletin de liaison des LPC, $n^{\circ} 203$, p. 121-123, 1996.

[3] Gambin M. - Le pressiomètre et la détermination de l'angle de frottement et de la cohésion d'un sol. Geoprojekt XXV Lat, 1952-1977 - Halin, 26 Wrzes- nia 1977.

[4] Monnet J., Khlif J. - Étude théorique de l'équilibre élastoplastique d'un sol pulvérulent autour du pressiométre. Revue Française de Géotechnique, n ${ }^{2} 67$, p. 4 12, 1994

[5] Hill. - The mathematical theory of plasticity, Oxford University Press, New York, 1950.

[6] Mènard L. - Mesure in situ des propriétés physiques des sols. Annales des Ponts et Chaussées, mai-juin. 1957.
[7] Wood D.M., Wroth C.P. - Some laboratory experiments related to the results of pressuremeter tests. Geotechnique, vol. 27, 2, p. 181-201, 1977.

[8] Withers N.J., Howie J., Hugues J.M.O. Robertson P.K. - Performance and analysis of cone pressuremeter in sands. Geotechnique, vol.39, 3, p. 433-454, 1989.

[9] Bolton M.D. - The strength and dilatancy of sands. Geotechnique, vol. 36, 1 . p. $65-78,1986$ 